\title{
Quaderni
}

QUADERNI Communication, technologies, pouvoir

\section{Des usages de la vie privée dans la (dé)légitimation politique}

\section{Fabrice d'Almeida}

\section{(2) OpenEdition}

\section{Édition électronique}

URL : http://journals.openedition.org/quaderni/488

DOI : 10.4000/quaderni.488

ISSN : 2105-2956

\section{Éditeur}

Les éditions de la Maison des sciences de l'Homme

\section{Édition imprimée}

Date de publication : 5 avril 2010

Pagination : 75-86

Référence électronique

Fabrice d'Almeida, "Des usages de la vie privée dans la (dé)légitimation politique », Quaderni [En ligne], 72 | Printemps 2010, mis en ligne le 05 avril 2012, consulté le 01 mai 2019. URL : http://

journals.openedition.org/quaderni/488; DOI : 10.4000/quaderni.488 


\section{$D$ ossier}

\author{
des usages \\ de la vie \\ privée \\ dans la \\ (dé)légitimation \\ politique \\ Fabrice \\ d'Almeida
}

À l'été 1914, l'attentat de Sarajevo passe presque inaperçu dans la presse française. Rares sont ceux qui mesurent la portée de cet événement survenu dans un État somme toute assez éloigné. Chacun espère que la tension balkanique retombera, comme par le passé. Non, la passion française est tournée vers un procès à sensation, celui d'Henriette Caillaux ${ }^{1}$. La femme de Joseph Caillaux, ministre des Finances démissionnaire, a, en effet, abattu de quatre coups de feu le directeur du Figaro, Gaston Calmette, qui menait contre son mari une terrible campagne de dénigrement depuis le début de l'année.

Lors des audiences, l'avocat de la défense, Fernand Labori, choisit d'orienter le procès vers la vie privée et de détourner les accusations politiques lourdes que portait Calmette. $\mathrm{M}^{\mathrm{me}}$ Caillaux aurait agi par peur que ne soit révélée sa liaison

Professeur

Université Panthéon-Assas, Paris 2 Institut français de presse - CARISM avec Joseph Caillaux, alors qu'il était encore marié à Berthe Geydan, sa première épouse. Elle craignait surtout que ne viennent au jour des informations concernant sa vie amoureuse.

Pourtant, le Figaro poursuivait un objectif politique. Calmette soutenait la politique de Poincaré et de Barthou qui tenaient pour une stratégie internationale ferme et combattaient la politique fiscale de gauche prônée par Caillaux. Les dirigeants de la droite modérée auraient ainsi poussé le directeur du journal à lancer la campagne de presse afin de fragiliser leur adversaire. La ruse de Calmette a consisté à trouver chez Berthe Geydan des documents qu'elle avait conservés en prétendant les avoir détruits. L'épouse délaissée cherchait ainsi à se venger de l'adultère que son mari lui avait fait subir pendant trois ans, avant de divorcer finalement en 1911. Les 
documents contenaient des remarques sur la politique intérieure et extérieure. Calmette les exploita en montrant la duplicité de Caillaux. Le ministre des Finances redoutait ces chroniques assassines et craignait surtout la publication des lettres montrant ses tractations avec l'Allemagne, lettres qui auraient pu lui valoir l'accusation de trahison. L'attaque sur la vie privée portait sur le manque de dignité d'un homme d'État qui signait ses lettres d'amours «Ton Jo». Le surnom fut repris par le Figaro et par l'Action Française, le quotidien monarchiste et raciste. Le 13 mars 1914, Calmette cita une lettre où Caillaux se vantait d'avoir attaqué l'impôt sur le revenu en feignant de le défendre, preuve manifeste de son hypocrisie. La violation de l'intimité servait donc à détruire l'image d'intégrité que Caillaux tentait de maintenir comme homme de gauche. Mais elle était nettement mélangée à des motifs politiques, signe que l'histoire d'adultère avait peu d'importance. Quelques années plus tôt, d'ailleurs, une tentative strictement privée contre Clemenceau avait totalement échoué devant la Chambre, qui considérait que les affaires amoureuses ne regardaient que les intéressés et qu'elle n'avait pas à lever une immunité parlementaire pour ces questions. Les députés s'amusaient même entre eux des exploits de leurs collègues. L'affaire Caillaux était donc une exception et une nouveauté et, n'eut été l'intervention des deux épouses Caillaux, elle serait sans doute restée confinée sur le terrain politique et aurait été vite occultée par la montée de la crise qui conduisit à la déclaration de guerre de l'Autriche à la Serbie au lendemain du verdict d'acquittement pour Henriette Caillaux, le 29 juillet 1914.

Près d'un siècle plus tard, les images de Ceci- lia Sarkozy sortant d'un hôtel avec son amant Richard Attias paraissent anodines ${ }^{2}$. Elles déclenchent une polémique sur les empiètements sur la vie privée des hommes publics mais, pourtant, ne suscitent pas une véritable exploitation politique. Mieux, le ministre de l'Intérieur utilise le ressort de cette crise de couple pour laisser entendre qu'il a mûri, " changé », appris à vivre en somme. De cette manière il évite le débat politique, tout en restant un politique ${ }^{3}$. Ses discours politiques jouent sur le non-dit et ses adversaires n'osent employer l'argument pour en tirer des conséquences sur l'incapacité à veiller sur sa famille. Sans doute redoutent-ils d'être confrontés à un danger similaire. Nul n'ose véritablement engager sa crédibilité politique sur la vie privée et donc, logiquement, l'usage de la vie privée dans les processus de délégitimation reste difficile pour qui voudrait porter le débat sur ce seul terrain. Et l'affrontement entre les acteurs politiques et la presse qui s'attache à leur vie privée se fait en France davantage sur le terrain judiciaire que politique.

La comparaison à un siècle de distance entre $\mathrm{M}^{\mathrm{me}}$ Caillaux et $\mathrm{M}^{\mathrm{me}}$ Sarkozy est éclairante. $\mathrm{M}^{\mathrm{me}}$ Caillaux prit sur elle le meurtre de celui qui avait brisé le tabou et décharge son mari de cette pression, car Caillaux n'avait guère de moyens d'action face à un adversaire qui se dérobait et ne lui aurait certes pas donné l'excuse d'un duel ou d'une affaire d'honneur pour entraver la campagne de dénigrement. À l'inverse, Nicolas Sarkozy a introduit seul sa vie privée dans le débat, mais agit de lui-même pour empêcher de nouveaux débordements en menaçant les journaux qui oseraient poursuivre sur ce terrain. Alain Genestar, le directeur de Paris-Match, en a 
fait l'amère expérience, lui qui dut démissionner après la publication de photographies en couverture de son magazine montrant Cecilia Sarkozy et son amant ${ }^{4}$.

L'objet de cet article est précisément d'essayer de comprendre les rapports que la vie privée entretient avec la légitimité politique. Quand la vie privée est-elle devenue l'objet d'un intérêt particulier pour les entrepreneurs partisans ; quand et comment a-t-elle fait l'objet d'usages critiques envers des hommes d'État; quels effets durables ou éphémères la délégitimation par la vie privée peut-elle avoir sur le fonctionnement de la société politique ? Ces questions sont d'ailleurs à conjuguer différemment selon que ce sont des pays européens ou d'autres cultures qui sont sollicitées.

Le cadre ici interrogé reste en tout cas celui de la politique de masse. Il invite à penser les comportements sur la scène publique dans un cadre différent de ce qu'aurait pu être une monarchie $\mathrm{du} \mathrm{XVII}^{\mathrm{e}}$ ou du XVIII ${ }^{\mathrm{e}}$ siècle, quand la distinction entre vie publique et vie privée était impossible. La politique étant alors incarnée, la personne juridique incluait tous les actes institutionnels ou pas. Aussi tenterons-nous, dans un premier temps, de revenir sur l'invention de la vie privée et de ses rapports avec la politique de masse, avant d'observer comment s'est imposée une première conception supposant que l'intimité ne soit pas dévoilée dans l'action publique. Puis l'étude abordera la façon dont ce cadre s'est modifié sous l'effet d'un principe de publicité généralisé superposant vie privée et vie publique. Le sujet est vaste, et ne saurait être traité en quelques pages. Il s'agit dans cette brève présentation de l'approcher à l'aide de quelques exemples afin de souligner la persistance de tabous et de limites qui signalent les contours de la démocratie d'opinion'.

\section{L'affirmation de la vie privée}

La question de la vie privée a émergé progressivement à partir de la fin du XVII ${ }^{\mathrm{e}}$ siècle. Sans doute sous l'effet du premier transfert de sacralité vers l'État absolutiste se fait jour la demande d'une distinction entre un espace pouvant être travaillé par la loi et un autre qui pourrait s'en extraire, renvoyé à l'intimité d'une croyance constituant progressivement le terreau du sujet ${ }^{6}$. Cette distinction est reprise notamment par ceux qui, tel Montesquieu, définissent un espace du droit qui s'arrête aux mœurs et donc suppose que l'État ne puisse empiéter sur les prérogatives des familles? À travers la structuration de l'opinion publique alors qu'entre en crise le modèle absolutiste, puis que sont reconnus les droits individuels au regard des sociétés politiques, comme l'expriment les diverses déclarations des révolutions civiques, de la constitution corse de 1755 à la déclaration française des droits de l'homme en 1789, se définit la rupture entre une scène publique où s'exprime la communauté des citoyens et une sphère privée où seraient gérées les seules relations humaines. Cette rupture met un terme à la logique qui avait présidé à l'établissement de la société de cour, à savoir un règlement de la chose privée sur le modèle de la chose publique, car les deux éléments étaient superposés dans la personne du roi. La disjonction entre fonction et personne produite par la démocratie libérale nécessite la définition théorique puis l'entrée en pratique de deux espaces distincts. Sans doute cette situation est-elle renforcée par le rejet des femmes de la 
citoyenneté qui supposait donc la reconnaissance d'un lieu particulier où elles exerceraient leur magistère, la maison, tandis que le forum serait la place des hommes. Une telle logique a été décrite en partie dans le concept de division des tâches entre hommes et femmes telle que l'a décrit Joan $\mathrm{Scott}^{8}$. Aussi le XIX ${ }^{\mathrm{e}}$ siècle voit-il le renforcement de la protection de la vie privée et la solidification de son rôle comme coulisse indispensable pour les hommes publiques.

Première au monde à exprimer cette dichotomie et à créer les conditions de sa reconnaissance par les tribunaux, la loi française de 1868 sur la presse crée une sorte de droit géométrique (Polybe) protégeant la vie privée. Plus un individu jouit de la puissance publique, moins sa vie privée est protégée. L'empereur Napoléon III pouvait ainsi voir sa famille exposée et ses mœurs critiquées alors qu'un obscur fonctionnaire serait fondé à poursuivre le journal qui exposerait ses proches au regard public. Le droit à la vie privée est ainsi reconnu. Il s'inscrit dans la perspective ouverte par la lutte contre la diffamation telle qu'elle est connue en Angleterre depuis le XVIII ${ }^{\mathrm{e}}$ siècle : la presse ne doit pas critiquer sans preuve et le dévoilement des mœurs d'autrui frise la calomnie. Pourtant, il faut bien le dire, les journaux exercent parfois un chantage sur les députés quand ils détiennent une information croustillante ${ }^{9}$. La dignité des hommes politiques est donc partiellement protégée de la divulgation de détails trop frivoles. Cette situation s'explique par leur rôle particulier dans des sociétés qui conservent le culte de la nation dont les gouvernants sont les dépositaires. Ce sont d'ailleurs eux qui décident de la guerre et, partant, de la vie ou de la mort des citoyens. Ce relatif attachement à un statut exorbitant du droit privé des personnes publiques est un des éléments constitutifs de l' « aristocratie élective », dont parle Bernard Manin, au sens où les électeurs admettent que la supériorité personnelle prêtée aux élus peut leur épargner une dégradation trop visible $^{10}$. Au début du $\mathrm{XX}^{\mathrm{e}}$ siècle donc, la vie privée est relativement protégée, mais chacun sait que cette protection est fragile. Elle dépend largement de la popularité et de la personnalité des élus. La dignité ne vaut que si elle n'est pas souillée, que si l'honneur peut être défendu, au besoin les armes à la main, dans un due ${ }^{11}$. Mais une femme ne peut se défendre ainsi. Elle est tributaire d'un mari qui lui-même ne peut, sans risque, se réfugier derrière des affaires d'honneur. D'où les craintes de $\mathrm{M}^{\text {me }}$ Caillaux qui voit le combat politique changer de nature et risquer d'entraîner son mari dans une tourmente.

Car, depuis l'affaire Dreyfus, la presse ne respecte pas toujours les limites que lui imposaient les hommes politiques. Des extrémistes de droite comme Drumont veulent prouver que la race conditionne les choix officiels ou personnels. Les juifs sont, selon eux, néfastes partout et leur griffe se retrouve dans tous les drames. Une telle logique conduit à prendre en compte d'autres aspects de la vie jusqu'alors laissés au seuil de l'espace public. N'a-t-elle pas poussé à jouer sur l'émotivité du public pour attiser la haine de Dreyfus après le suicide du colonel Henry? N'est-ce pas tout l'enjeu de la quête pour la veuve et les enfants de l'auteur du « faux patriotique »? Les scandales effrayent une partie de la classe politique qui répugne à ces pratiques nouvelles de critiques outrancières et d'injures racistes. La Première Guerre mondiale arrive à point, qui renforce la distinction entre le public et le privé, 
en posant que la souffrance nécessite son intimité, son quant-à-soi, pour ravaler ses larmes. La dignité des élus est restaurée par le sang.

Pourtant, la guerre a deux effets collatéraux. D'abord elle introduit des conduites moins strictes que celles issues des traités de bonnes manières du siècle précédent. Elle autorise un style moins distingué, plus proche du peuple, le brassage social a été profond pendant les années de conflit. Ensuite, la Grande Guerre pousse à valoriser une conception de l'homme politique qui doit partout avoir la même conduite, et les mêmes idées. Elle renforce l'idée pernicieuse qu'il faut vivre conformément à son idéologie. Cette question touche particulièrement les dirigeants qui cherchent une légitimité par l'action et construisent leurs actes comme de véritables gestes de propagande. Agir, pour Mussolini, Hitler, Salazar, Staline ou Franco, c'est dire. Plus que les démocrates, tels Blum ou Amendola voire Nenni et Alcala Zamora, ils ont compris le poids des signes et jouent de leur comportement pour bâtir une légitimité nouvelle. Les démocrates osent moins impliquer leur vie privée que les dictateurs.

\section{Charisme, totalitarisme et vie privée}

Les chefs charismatiques vivent sous la pression d'une contrainte dont ils fixent eux-mêmes les contours et qui leur impose une présentation de soi particulière. Ils inventent et politisent des conduites, les intègrent dans leur agenda et leur confèrent un sens politique qu'elles ne possédaient pas originellement ${ }^{12}$. L'anthropologue Luc de Heusch avait souligné les rapports que le charisme entretient avec la notion romaine de celeritas, la capacité qu'a le chef victorieux de créer un système de légitimation qui repose sur sa fortune personnelle ${ }^{13}$. Son culte se substituait ainsi à celui des institutions, à la religion civique, qui exigeait de ses représentants ce que Georges Dumezil appelait la gravitas, une attitude mûre et digne $^{14}$. Le même basculement s'observe quand les institutions italiennes, allemandes et russes tombent dans le fascisme, le nazisme et le stalinisme ${ }^{15}$. La celeritas étant liée à la personne du chef, elle est présente dans tous ses actes, y compris les plus anodins. Précocement, les dirigeants construisent une fiction de vie privée à usage de la presse afin de laisser au peuple l'impression qu'ils sont conformes à l'exigence du nouveau culte dans la totalité des domaines que recouvre une existence humaine. Voici Mussolini sélectionnant des images de ses loisirs tel un aristocrate, présentant des photos de famille et mettant en scène le mariage de sa fille Edda avec le comte Ciano dans la grande tradition des familles princières. Il cache un temps sa liaison avec Claretta Petacci qui pourrait offenser la morale des catholiques mais la laisse entrevoir afin de renforcer le culte de sa virilité ${ }^{16}$. Salazar, Hitler et Staline jouent sur une autre image. Ils feignent de n'avoir d'autres liaisons amoureuses qu'avec l'État et exaltent leur célibat comme preuve de leur dévouement. La propagande salazariste s'appuie sur son veuvage pour le présenter en digne patriarche dévoué à la cause commune. Le mot d'ordre de Hitler résume bien cette posture : « Mein Braut ist Deutschland» (ma fiancée est l'Allemagne). La légende communiste de la lumière du Kremlin qui reste allumée toute la nuit car Staline ne dort jamais en est un avatar.

La propagande hitlérienne valorise la vie privée du leader bien au-delà de la question de son 
rapport avec les femmes. Ses loisirs sont mis en scène dans des ouvrages illustrés qui sont diffusés à plusieurs centaines de milliers d'exemplaires et dont les images sont aussi données en cadeau promotionnel dans les paquets de cigarettes de la firme Reemtsma ${ }^{17}$. Le nazisme ne s'arrête pas à cet usage convenu de la vie privée dans l'écriture d'une légitimation de la personne du leader ${ }^{18}$. Il emploie à plusieurs reprises la vie privée comme argument à l'encontre de ses adversaires. Après les liquidations de la direction de la SA et de dirigeants modérés, le ministère de la propagande pousse la presse à publier des articles dénonçant la déviance homosexuelle de Röhm et de ses proches. Le même motif est utilisé en 1938 contre le général von Fritsch contraint de démissionner. Quant au général von Blomberg, il se retrouve obligé de quitter ses fonctions lorsque, habilement, la presse révèle que sa femme avait travaillé dans le salon de massage de sa mère et laisse entendre qu'elle est une ancienne prostituée ${ }^{19}$. Le procédé est tel que des rumeurs circulent sur les proches d'Hitler dont le public suppose qu'ils sont tenus par des secrets terribles. La femme de Julius Schaub, l'ancien chauffeur devenu patron des adjoints de Hitler, serait ainsi l'objet du chantage exercé sur son mari car elle aurait aussi été une prostituée.

Descendue à ce niveau de polémique, la propagande pousse les opposants au régime à utiliser des arguments du même genre pour tenter de discréditer Hitler et les dirigeants nazis. Strasser, dont le frère a été assassiné en 1934, écrit ainsi un essai vengeur où il évoque Hitler comme homosexuel; les services secrets américains chargent des psychologues d'établir un profil aussi sordide que possible d'où il ressort que le Führer serait coprophage et adorerait voir les femmes uriner; même d'anciens amis transfuges aux États-Unis à la veille de la guerre trouvent le moyen d'écrire que le dirigeant n'aurait pas de vie sexuelle et sublimerait ses excès de libido dans la consommation immodérée de pâtisseries horriblement sucrées ${ }^{20}$. En somme, le nazisme a largement contribué à faire entrer la vie privée dans les instruments de montage d'opération de délégitimation. Sans doute parce que, comme le soulignait Hannah Arendt, les États totalitaires tendent à écraser la vie privée ${ }^{21}$.

Cette émergence sur la scène publique et la contamination qu'elle engendre dans les démocraties restent toutefois limitées au lendemain de la Seconde Guerre mondiale. Il faut attendre les années 1960 pour que la vie privée connaisse un usage plus lourd de conséquences, tant en matière de légitimation qu'en fait de délégitimation. Cela constitue un retard important par rapport aux États totalitaires qui n'ont cessé après 1945 de parfaire leur technique de dénigrement et de désinformation sur la base des mœurs et de la vie privée. L'aveu d'Arthur London ou les campagnes antisionistes de la Pologne des années 1960 s'appuient largement sur cette question pour discréditer les opposants. Dans les démocraties, le phénomène évolue plus lentement sans doute parce que, là, la séparation nette entre vie publique et vie privée est reconstruite comme une garantie pour les droits des citoyens à agir dans l'espace public. Quelques affaires émaillent cette évolution comme le cas du ministre britannique John Profumo contraint à démissionner à cause de sa liaison avec une jeune actrice dont il partageait les faveurs avec le conseiller militaire de l'ambassade de l'Union soviétique. 


\section{Critiques démocratiques des turpitudes privées}

Lentement d'abord, puis plus rapidement, l'idée de la cohérence entre vie publique et vie privée des élus revient. Sans doute le mouvement des idées a-t-il été à l'origine de cette construction. D'abord parce que le recul des idéologies rendait moins pressant un engagement qui dépassait les petites affaires personnelles. Ensuite, les conditions techniques de la télévision, de la vidéo et de la photographie, avec des téléobjectifs plus puissants, rendent plus mobiles les prises de vues et facilitent une observation des sujets à leur insu. S'ajoute à cela un principe de publicité qui suppose que les positions partisanes correspondent à des manières d'être et que les plus petits symboles de la vie quotidienne soient susceptibles de signifier une option politique. Dans cette logique, les candidats sont amenés à présenter aux électeurs une fiction de vie privée qui doit reproduire les choix du programme politique. La correspondance devient ainsi étroite entre agir en politique et être dans la vie. Cette naturalisation de la politique place donc tous les secteurs de l'existence sous la loupe des médias, dès le moment où une personne atteint un niveau de notoriété élevé. Loin de provenir du seul désir des hommes politiques ou des journalistes, cette situation provient d'une spirale d'information dont le moteur est la soif inextinguible des électeurs de rassembler des connaissances sur ceux qui les entourent au point de confondre parfois le principal et l'accessoire.

Dans ce contexte, la délégitimation par la vie privée prend une coloration spécifique. Elle ne résulte pas forcément d'une manipulation politique comparable à celle que Barthou et Poincaré menaient en sous-main contre Joseph Caillaux. Elle provient simplement du travail de dévoilement des médias qui mécaniquement produit des données négatives dans la masse d'images, de textes, de reportages, d'enquêtes et de confidences qu'ils recueillent. L'exemple de Nicolas Sarkozy et de ses problèmes de couple en témoigne puisque les premières images dévoilant les relations entre la femme du ministre et son amant ont été publiées dans la presse étrangère ${ }^{22}$. Cette dernière a fait peser une pression sur la presse française.

Mis au déf, les journalistes de l'hexagone avaient l'air d'être inféodés au ministre de l'Intérieur s'ils ne passaient pas à la publication alors que leurs confrères étrangers proposaient les textes et les images sur Internet. La crise médiatique laissait ouverte la question de la légitimité avec l'idée sous-jacente de savoir si un homme qui peinait à gérer son ménage serait capable de diriger la France. Nulle action d'un adversaire n'était donc à la source de cet événement.

À gauche, le dirigeant socialiste Arnaud Montebourg a prétendu être victime d'une manipulation similaire de la presse, quand il fut présenté dans le très populaire magazine Gala, devant sa belle maison de campagne, tel un Gentleman Farmer et un Play Boy ${ }^{23}$. Il déclara avoir été photographié au téléobjectif, sans avoir été prévenu. Il souhaitait porter plainte, comme le fit Nicolas Sarkozy. Dans les deux cas, toutefois, l'affaire n'eut pas une portée durable en matière politique.

Arnaud Montebourg fut pourtant à l'origine d'une situation inédite concernant la vie privée de Ségolène Royal quand il déclara dans une 
émission de télévision que le principal problème de la candidate socialiste n'était autre que « son compagnon ». Cette mise en cause lui valut d'être suspendu pendant un mois de sa fonction de porte-parole. Sans doute s'agissait-il d'une maladresse, mais le mot visait le secrétaire du PS et reflétait la tension existant entre le parti et le cabinet de campagne de $\mathrm{M}^{\mathrm{me}}$ Royal. L'humour était oublié, comme si l'intrusion dans la vie privée n'était pas admissible pour émettre une critique à l'encontre de son propre camp. La vigueur de la réaction était à la hauteur des réactions de la presse stimulée par la députée sarkozyste Nadine Morano. Dans cette occurrence, les médias trouvaient leur compte à réprimer le débordement d'un homme politique car ce geste servait leur objectif de gagner en audience et le désir des membres de l'UMP de semer la discorde au sein de l'équipe de gauche.

L'épisode permet de souligner combien les liens entre le monde médiatique et le monde politique introduisent de biais dans la compréhension des mécanismes de délégitimation à l'œuvre. Cette question est d'ailleurs relativement ancienne car, pour dénoncer les turpitudes ou les caractéristiques de la vie privée d'une personne, il faut les connaître ou du moins en avoir des preuves. Or, ce sont souvent des proches qui sont capables de trouver les points les plus sensibles, pour lancer une opération de déstabilisation. Quand Jacques Chirac rompt en 1976 avec Valéry Giscard d'Estaing, il utilise le penchant de ce dernier pour le décorum afin de le présenter comme une sorte de malade mental se prenant pour un roi et disposant d'un trône à domicile ${ }^{24}$. L'entourage du Premier ministre laisse ainsi filtrer vers la presse une image volontairement caricaturale afin de faire porter au président la dégradation des rapports entre les deux hommes afin que Chirac soit le seul bénéficiaire de la chute prématurée du gouvernement, avant l'échéance des municipales de 1977 et des législatives de 1978.

En Italie, ces usages tactiques de la dénonciation médiatique sont courants. Cette violence critique va plus loin que la question du domicile ou de la famille. Les attaques contre Silvio Berlusconi en sont un bon exemple : moqué pour sa villa d'Arcore avec son mausolée monumental, critiqué pour ses relations personnelles avec des hommes pour le moins corrompus, le président du conseil est bientôt brocardé pour sa taille, ses implants capillaires ou son lifting. Ces accusations furent suffisamment répétées pour qu'il décide par exemple de montrer l'intérieur de ses chaussures afin de contredire les accusations. Il se sentit aussi obligé de justifier les retouches visuelles sur ses affiches électorales, en 2001. Derrière le débat sur son corps vieillissant se devine le désir d'une vérité partisane supposant une transparence telle que le corps du chef devrait être nu. Le conte d'Andersen dit pourtant que, lorsque le roi est nu, le peuple doit feindre de n'en rien voir, et que seul un enfant manque à ce point d'éducation qu'il ne reconnaît pas l'impératif de discrétion.

\section{Des frontières politiques et une intimité en question}

Cette évolution reflète le changement de statut de la politique dans la société. Naguère distincte des autres activités sociales car dépositaire d'une sacralité et lieu de construction de la transcendance nationale, elle se coule désormais dans le moule des métiers publics. Elle est donc aussi 
sensible au glamour que les stars de cinéma et à des processus de connivence que connaissent bien les industries du show business. La mise en forme de la vie privée des hommes publics évoque celle de toutes les célébrités. Elle subit les aléas de l'opinion et les modes. Elle établit une hiérarchie selon une cote de popularité qui répond à des critères indépendants de ceux concernant les décisions politiques. Ainsi l'humour est-il devenu une clé du vedettariat politique là où auparavant l'agilité doctrinale tenait lieu de viatique.

Les scandales les plus graves sont sans doute survenus aux États-Unis où un candidat comme Gary Hart n'a pu poursuivre sa course présidentielle quand a été révélée sa liaison avec une call girl ou lorsque le président Bill Clinton fut contraint de se soumettre à une commission d'enquête dirigée par un procureur fédéral afin d'éclaircir les relations qu'il avait eues avec une de ses stagiaires. Précisons toutefois que si le coût institutionnel fut élevé, le coût politique pour Clinton fut relativement faible et que sa présidence a été suffisamment forte pour que cet épisode renverse désormais la pression sur la vie privée des hommes et des femmes politiques américains. Ils sont encore présentés avec une apparence de glamour, mais le débat sur leur vie privée semble passé au second plan. Importent davantage les positions politiques et les collusions affairistes. Sans doute est-ce un effet de la guerre en Irak qui a modifié la hiérarchie des priorités et un retour à la paix pourrait corriger cette tendance.

L'attention sur la personnalité et la starification des dirigeants politiques ne doit toutefois pas laisser croire que la question de l'empiètement sur la vie privée ne se joue que dans des attaques sur la sexualité. L'intimité est violée aussi sur des terrains d'autant plus sensibles qu'ils sont couverts par des réserves professionnelles, tel le secret médical.

La question de la santé est, de fait, un des lieux où les indiscrétions sont le plus fréquemment recherchées et où les révélations produisent des effets violemment délégitimants. L'annonce du cancer de François Mitterrand ${ }^{25}$ fut la confession d'un secret trop lourd à cacher. Les bulletins de santé du président Chirac après son attaque cérébrale ont laissé beaucoup de doutes sur son aptitude à gouverner. Ces deux épisodes ont fragilisé la position de ces dirigeants sur l'échiquier politique. La dégradation physique des gouvernants est trop souvent traitée comme une anecdote alors qu'elle engage la représentation du pouvoir et la croyance en sa capacité d'action. Le risque de mort ou de maladie est une expression directe de la fin en politique. Les atteintes physiques ont leurs tabous.

Non moins empreinte de mystère est la question de la fragilité mentale d'un individu. Elle peine à être révélée et ouvre une telle inquiétude que le public ne peut la regarder en face. La mort volontaire ou l'internement pour défaillance psychique sont de ces événements qu'un pouvoir tente de masquer ou de détourner afin d'éviter qu'à travers eux une menace ne pèse sur les proches politiques de la victime. Le gaullisme cacha ainsi les séjours d'André Malraux alors ministre de la Culture dans des établissements spécialisés quand il s'effondrait. Les mitterrandiens expliquèrent le suicide de François de Grossouvre et celui de Pierre Bérégovoy par une faiblesse particulière de leur caractère et de leur situation ${ }^{26}$. Mitterrand lui-même attribua le suicide au harcèlement des 
journalistes, refusant de prendre en compte le relatif abandon qu'avait pu ressentir son ancien collaborateur. Car l'amitié en politique n'est pas seulement affaire publique. Elle est aussi vie quotidienne et partage de situation familiale et de réconfort dans les moments difficiles. Rarement, la presse valorise cette question sinon quand un drame se produit, tel le suicide de Philippe Habert, le gendre de Jacques Chirac, retrouvé seul un jour de 1993, dans son appartement, mort comme frappé de disgrâce. Prudent, le Figaro, dont Habert était pourtant le chef des études politiques avait fermé toute lecture institutionnelle, laissant le deuil se poursuivre, en respectant la famille du chef du RPR. La limite de la politique fluctue donc autant que celle de la vie privée. L'interrogation sur ses usages délégitimants suppose une casuistique fine qui ne s'intègre pas toujours dans les grandes catégories d'interprétation.

\section{Retour sur la légitimité}

Tournée vers l'espace public, la légitimité politique repose d'abord sur la question centrale du vote, de la délégation de pouvoir et de la représentation. Même les États totalitaires ont vécu de cette fiction d'élection et de légalité que conférait tout un étagement de scrutins contraints par des règles absurdes dont l'objectif était d'essayer d'éviter les surprises du suffrage. Pourtant, là aussi, l'opinion produisait ses rumeurs et ses contradictions qui pouvaient faire tomber un dirigeant en disgrâce, voire engendrer sa liquidation.

La réflexion sur la délégitimation par la vie privée vaut cependant particulièrement dans la séquence qui s'est ouverte depuis les années 1980. Cette dernière voit une montée en puissance de la dé- mocratie d'opinion. Ce contexte se traduit par une sélection des candidatures politiques au gré des résultats de sondages, et leur publication par de puissants outils médiatiques. Les mouvements de l'opinion sont, dès lors, sensibles à d'autres formes que la légitimité du vote puisque, par la manifestation d'un mécontentement et l'expression d'une affection, une fraction du corps électoral peut valider un candidat. Ici, la question de la délégitimation ne dépend donc plus de l'émission formelle, constitutionnelle, de l'opinion mais d'un processus labile sur lequel même les hauts responsables ont peu de prise. Les voici contraints de suivre des tendances et des modes qui leur échappent. Le théâtre du pouvoir ne parvient pas à restreindre la sphère observée par le public. Les critères de choix ne correspondent plus aux exigences des programmes voire aux besoins des électeurs. Dès lors, les élus tentent d'anticiper sur la demande en proposant une vision tronquée de leur existence, incluant toutes sortes de caractéristiques officielles et officieuses, publiques et intimes. Ils décrivent leur hobby, leur famille, montrent leur intérieur et leur animal domestique et n'hésitent pas à s'inventer des loisirs et des passions qui leur donnent une originalité.

Cette attitude déplace à son tour la question de la légitimité politique du vote vers celle de sa recevabilité. Elle crée une catégorie d'hommes politiques particuliers qui, sans jouir d'un véritable pouvoir, possède une capacité d'influence en raison même de sa compréhension de la sphère médiatique et de sa production de stars. L'espace de la classe politique voit ainsi son centre de gravité se déplacer des lieux de décision et de définition de la loi, vers les espaces de fabrication de l'opinion et de construction de la lecture 
populaire du monde. Les individus performants au regard des sondages, et des médias, tendent ainsi à remplacer les techniciens du débat parlementaire. Ce faisant, ils substituent aux formes de légitimité issues des rituels républicains des cérémonies d'intronisation numérique. Ils érigent une frontière nouvelle dans la distribution entre espace public et espace privé en y superposant une distinction entre des lieux confidentiels et des lieux ouverts. Commence ainsi une crise institutionnelle puisque les coulisses des ministères ou du parlement sont dénoncées comme des refuges partisans secrets, alors même qu'elles répondent aux exigences constitutionnelles. Au contraire, les loges des studios de télévision, filmées par les caméras, sont vantées tels des lieux ouverts, alors qu'aucune règle officielle ne les concerne. Ces inversions illustrent la confusion actuelle. Elles montrent que la protection de la vie privée des hommes publics n'est pas une question de défense de privilège mais qu'elle engage le fonctionnement des démocraties.

Naguère, Georges Balandier décrivait la théâtralité du pouvoir ${ }^{27}$. Aujourd'hui, la scène a disparu. Les acteurs politiques paraissent nus. Le souverain sans couronne, ni vêtement, se promène sous les rires et les quolibets d'un peuple qui a oublié qu'un peu de discrétion ne nuit pas au gouvernement. L'élu feint de croire qu'il règne encore, car il possède des armes et voit dans le sabre l'ultime ressource de sa grandeur. Dans les crises, les violences et la guerre, il retrouve sa dignité. Ce sursaut de conscience le ramène à la noblesse de sa tâche, quand les temps sont rudes aux hommes de bonne volonté. Il comprendra bientôt qu'un nouvel adage s'impose pour parer aux catastrophes : moins paraître et mieux être.
N

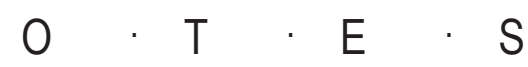

1. Jean-Claude Allain, Joseph Caillaux, Paris, 2 vol, 1978-1981.

2. Paris-Match, août 2005.

3. Erik Neveu, «De l'art (et du coût) d'éviter la politique. La démocratie du talk-show version française (Ardisson, Drucker, Fogiel)», Réseaux, 118, 2003, pp. 128-129.

4. Olivier Costemalle, "Paris Match : Genestar s'en prend à Sarkozy », Libération, 17 novembre 2006.

5. Sur la notion de démocratie d'opinion, voir Bernard Manin, Principes du gouvernement représentatif, Paris, Flammarion, 1996.

6. Marcel Gauchet, Le désenchantement du monde, Paris, Gallimard, 1985.

7. Montesquieu, L'esprit des lois, Paris, Gallimard, 2 vol., éd. 1995.

8. Joan Scott, La citoyenne paradoxale, Paris, Albin Michel, 1998.

9. Marc Martin, «Publicité, gestion et politique rédactionnelle », in Fabrice d'Almeida, La question médiatique, Paris, Seli Arslan, 1997, pp. 171-180.

10. Bernard Manin, Principes du gouvernement représentatif, op. cit.

11. Jean-Noël Jeanneney, Le duel - Une passion française, Paris, le Seuil, 2004.

12. Fabrice d'Almeida, La politique au naturel. Comportement des hommes politiques et représentations publiques en France et en Italie du XIXe au XXIe siècle, Rome, École française de Rome, 2007, p. 138 et sq.

13. Luc de Heusch, Rois nés d'un cœur de vache, Paris, Gallimard, 1982.

14. Georges Dumézil, La religion romaine archaïque, Paris, Hartmann, éd. 2000.

15. Ces catégories sont à discuter dans le cadre 
proposé par Emilio Gentile, Le religioni della politica, Roma, Laterza, 2000.

16. Voir entre autres Pierre Milza, Mussolini, Paris, Fayard, 1999.

17. Voir Heinrich Hoffmann, Hitler wie ihn keiner kennt, Berlin, éd. 1935 ; et du même, Hitler Abseits vom Alltag, Berlin, éd. 1937.

18. Pour tout ce qui suit, voir Fabrice d'Almeida, $L a$ vie mondaine sous le nazisme, Paris, Perrin, 2006.

19. Insitut für Zeitgeschichte, dossier Göring, interrogatoire.

20. Voir notamment Walter C. Langer, The Mind of Adolf Hitler, New York, Basic Books, éd. 1972.

21. Hannah Arendt, Les origines du totalitarisme, Paris, Gallimard, Quarto, éd. 2002.

22. Notamment dans le Matin (Suisse). La vie privée de Sarkozy débordait alors au point que le Nouvel Observateur (le 10/11/2005) révélait l'identité de sa nouvelle compagne (Anne Fulda, depuis occultée) qui devrait faire en novembre 2005 un entretien avec France Soir.

23. Daniel Bernard, «Le play-boy de Ségolène », Gala, 13 septembre 2006.

24. Catherine Nay, La double méprise, Paris, Grasset, 1980.

25. Claude Gubler et Michel Gonod, Le grand secret, Paris, Plon, 1996.

26. Roger Faligot et Rémi Kauffer, Éminences grises, Paris, Fayard, 1992.

27. Georges Balandier, Le pouvoir sur scènes, Paris, Balland, 1989.
$R \cdot E \cdot S \cdot U \cdot M \cdot E$ La vie privée est devenue progressivement objet d'attention pour les médias et les leaders partisans. En montrant leur existence quotidienne, les femmes et les hommes politiques ont tenté d'affirmer la qualité intrinsèque de leur être. Une manière, en somme, de se donner une légitimité assise sur la supériorité de leur naturel. A contrario, les attaques sur la vie privée sont donc devenues un moyen aisé pour délégitimer une personne publique.

\section{Abstract}

Private life is nowadays a major way to make up and assert the political legitimity of a leader. By showing their everyday life, their family and their leisures and pleasures, politicians pretend to give the true nature of their being. Therefore is it easy for their opponents to delegitimate them, by intruding their privacy. 